\title{
Proactive Biobanking to Improve Research and Health Care
}

\section{Sreeja Sarojini, Andre Goy, Andrew Pecora and K. Stephen Suh*}

John Theurer Cancer Center, Hackensack University Medical Center, NJ 07601, USA

Keywords: Biobank; Tissue bank; Tissue repository; Personalized medicine; Targeted therapy; Cancer care

\section{Introduction}

Biobanking, the archiving of biological specimens from donors for use in research, has become an important resource for healthcare research, medical care, and drug discovery. The kinds of questions being addressed in contemporary medical research depend upon the availability of biological tissue samples along with associated clinical data including medical, genealogical, and lifestyle information [1]. International awareness of the need for biobanks has increased, in part, because of the growing promise of research related to the development of personalized medicine, especially in oncology (Figure 1). Identification and validation of disease-relevant biomarkers is essential [2] to developing diagnostic, prognostic, and therapeutic applications that will improve community health and protect the public from illnesses. For these purposes, high-quality and well-annotated tissue sample collections housed in specialized bio-repositories must be available. The single, most important roadblock in translational research is the limited availability of human tissue specimens; hence, outreach efforts to encourage and educate the public toward donating tissue samples and body fluids for biobanking is essential. Efforts to increased societal awareness and understanding of the needs and purpose of biobanks in healthcare research will contribute to increased trust and confidence of the community regarding biobanking. Robust community participation is a necessary component of any effort toward realizing the potential of biobanking. This article discusses many aspects of biobanking, the need for increased public awareness, general public misconceptions about donating samples, and the need for sustained funding to allow biobanks to achieve their goals and purposes.

\section{Why Biobanking?}

Discovery of new drugs and personalized medicine for the effective treatment of diseases promises to play an important role in community health. Personalized medicine depends on a series of accurate diagnostic tests, which accurately identifies patients for targeted therapies. For example, overexpression of HER2 (human epidermal growth factor receptor-type 2), which occurs in a subset of breast cancer patients, provides important prognostic information with respect to patient response to the drug trastuzumab [3]. To match the HER2 expression profile with the prognosis and, importantly, potential therapeutic options, researchers needed to evaluate HER2 expression among high quality biospecimens that were associated with specific clinical outcomes. Without high quality biospecimens from biobanks, novel therapies and personalized medicine would be difficult to achieve. Thus, biobanking plays an important role in advancing biomedical and translational research, and provides better opportunities for promoting community health through the collection and preservation of biological samples such as blood, tissues, and nucleic acids, which are then made available for use in research endeavors to discover disease-relevant biomarkers that can be used for diagnosis, prognosis, and predicting drug responses (Figure 1).

The ultimate aims of biobanks include promoting the health and well being of the community [4] at no individual cost or risk. The clinical significance includes protection against current and emerging complex diseases through the development of personalized medicine. A major goal of personalized medicine is the development of highly targeted therapies that minimize or eliminate side effects. To put biobanking on the map of our healthcare system, proactive participation is needed from medical institutions, especially community medical centers. Typically, more than $80 \%$ of the general population is treated in a community medical center setting, and not by major academic medical centers. Patients simply do not travel to large academic medical centers when they first experience discomfort or observe initial clinical symptoms. However, community hospitals normally have neither the infrastructure nor the commitment to support biobanking; thus, millions of valuable, leftover tissue specimens and other clinical samples are discarded. Programs are needed that allow the local clinical community to increase their level of participation either by establishing a local biobank or through collaborative arrangements with academic institutions for shipping and curating of locally acquired biospecimens. The establishment of the necessary infrastructure and commitment will lay the foundation for our future research and healthcare. As electronic medical record (EMR) systems are implemented worldwide, clinical data associated with biospecimens will be readily available to biomedical researchers. Just as our current health care system is the product of previous medical research and scientific awareness that allows us to identify and control many diseases, contributions in current medical research will provide the underpinnings of the healthcare system for future generations. This future requires participation, not only of researchers, but also of physicians, clinicians, policy makers (institutional and governmental), and the general public.

There are numerous challenges to implement community wide biobanking including barriers for participation of diverse racial/ ethnic populations, and cultural and linguistic barriers that must be addressed. Effective biobanking requires educating the community to reduce misconceptions about donating tissues. Patients at large do not necessarily have a clear understanding of tissue banking, and may be afraid to participate. For example, many believe that surgeons will excise extra portions of tissues from their body during the operation. Some believe that they will not receive optimal medical care if they do not participate, thus although they sign the consent, their participation is not strictly voluntary. They may decide only to donate blood and not tissue. The availability of matching tissue and blood/bodily fluid is invaluable for identifying molecular biomarkers that are associated

*Corresponding author: K. Stephen Suh, John Theurer Cancer Center Hackensack University Medical Center, 30 Prospect Avenue, Hackensack N 07601, USA, Tel: 201 336-8214; Fax: 201-336-8776; E-mail: ksuh@humed.com

Received January 10, 2012; Accepted March 17, 2012; Published March 09, 2012

Citation: Sarojini S, Goy A, Pecora A, Suh KS (2012) Proactive Biobanking to Improve Research and Health Care. J Tissue Sci Eng 3:116. doi:10.4172/21577552.1000116

Copyright: @ 2012 Sarojini S, et al. This is an open-access article distributed under the terms of the Creative Commons Attribution License, which permits unrestricted use, distribution, and reproduction in any medium, provided the original author and source are credited. 


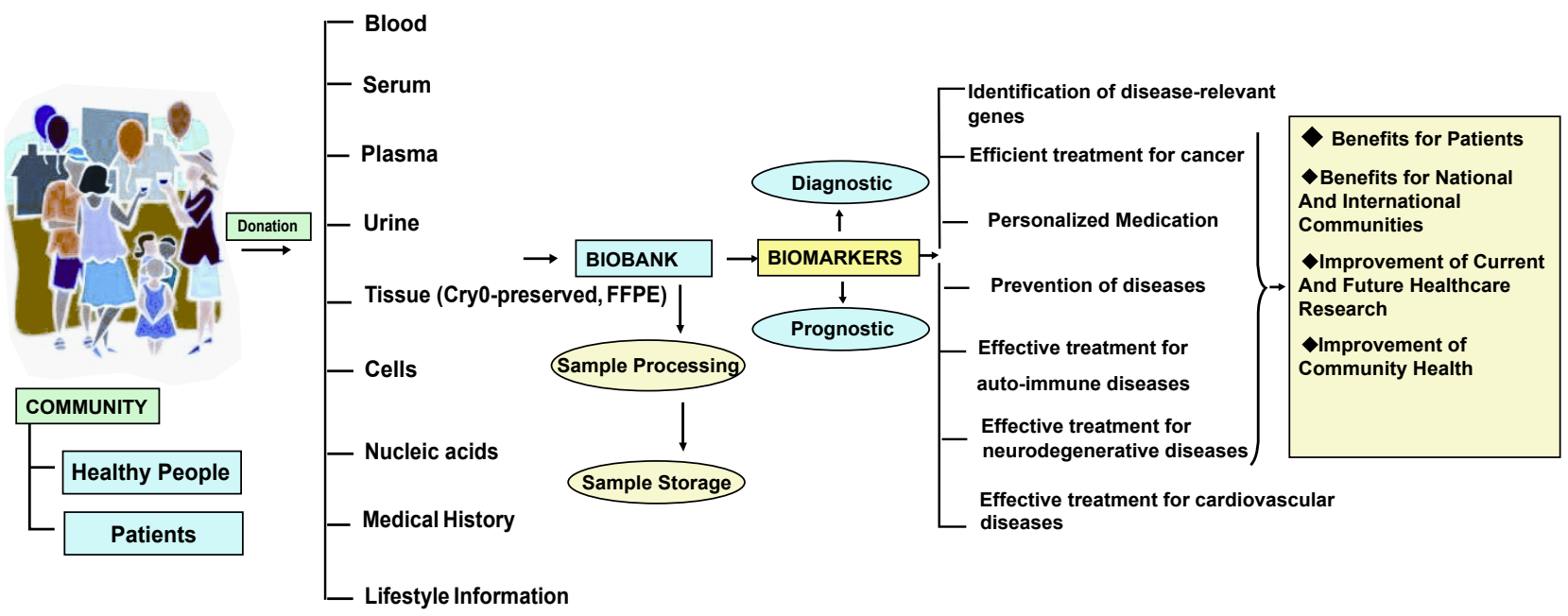

Figure 1: Biobanks improve community health by providing systematically preserved biospecimens to health care researchers for identifying diagnostic and prognostic biomarkers.

with the disease. These challenges can be reduced when biobanking concepts are taught in college level courses or in healthcare related meetings promoted by local communities. Community wide education brochures can be distributed by local governments working with hospitals and academic tissue banks to educate the population. The entry point to consent patients is another common barrier because the consenter must prescreen patients, and the patients typically are not focused on biobanking, but are concerned about their illness. Approaching patients when their mental and emotional state is subpar is not welcomed by risk management groups of any medical institution. The policies of the individual hospital or local government can become barriers to promoting effective biobanking; thus, institutional committees, IRB, and compliance departments must be work together to build an effective biobank that can contribute to healthcare.

There are different types of biobanks currently supporting medical research. Among them, population biobanks focus on identifying the country or geographic region of origin of healthy donors through germ-line DNA analysis. This can identify particular ethnic cohorts for study enrollment. The importance of population-based biobanks is that they merge genomics with public health-related research, by facilitating population-selective cohort studies [5]. Disease-oriented biobanks for epidemiological studies focus on identifying novel disease biomarkers through analysis of germ-line DNA or serum markers within a large group of collected samples. For example, cancer-oriented biobanks collect both normal and tumorous tissues from the same organs of individual patients, which permits identification of distinct tumorrelated biomarkers for further evaluation in clinical trials [6]. Diseaseoriented biobanks are usually hospital-based, and derive their samples from the patient population of the hospital, whereas population biobanks typically operate independently of hospitals, and donors are community volunteers rather than patients [7].

As described above, population-based biobanks procure biological samples from individuals randomly selected from a community, and these samples are archived along with associated patient demographic, lifestyle, and environmental data. Population-based biobanks are helpful in studying common diseases and their progression among community members, and for identifying potential predictive biomarkers of disease risk. Drawbacks of this approach include the need to accumulate large sample numbers and study groups, and lengthy follow-up periods (i.e., minimum 10-15 years) that are required. Follow-up information is critical to establishing a clinical outcome database that can be associated with disease biomarkers, which can be used for personalized medicine; without accurate clinical data and follow-up of outcomes, researchers cannot validate biomarkers to predict drug responses and efficacy. Preservation of sequential biological samples allows assessment of tissue changes over time, which is critical to understanding outcomes and age-related phenomena [8,9]. Population biobanks are generally large and are primarily supported by government funding (e.g., UK Biobank Limited, United Kingdom) and typically cover a large spectrum of diseases or genetic backgrounds. In contrast, diseaseoriented biobanks (e.g., tumor banks) include specimens from patient populations with specific disease types, and specimens are categorized based on diagnostic and treatment information. Many institutions, even at the level of department or individual principle investigator, can establish such small disease-specific tissue banks for research. Tissue banks are a popular specific format of disease-oriented biobanks, wherein tissues from patient donors (both normal tissue and diseased tissue) are collected with associated medical information. For instance, tissues are typically procured from cancer cases or from other organspecific systemic inflammatory diseases [10] after surgical removal of the lesions. Both types of biobanks ultimately advance research toward identifying disease-relevant biomarkers and genes by providing comprehensive sample collections to research scientists. The larger the number of high-quality tissue samples that are collected (both normal and diseased), the more powerful the biobank is in facilitating discovery of genetic mutations that cause disease or affect disease outcome [11].

Archived specimens are used for developing personalized medical treatment strategies in many illnesses, including cancer (Figure 1). Normally, these archived tissues, which are obtained from the pathology department of the institution and transferred to the biobank, are preserved either in formalin fixed, paraffin embedded blocks or in the format of frozen cassettes. Acquiring all necessary documentation and policy compliance assertions for the transfer of biospecimens from the pathology department to the biobank can create significant challenges, because all personal health information must be deidentified, and in most cases, the patients from which the archived 
samples were obtained were not consented. Most community hospital biobanks work directly with surgical and pathology departments to procure prospective biospecimens.

Disease-associated biomarker identification is significant; these markers are the molecular products, i.e., proteins, DNA, RNA, metabolites, or fatty acids [12], that are selectively or differentially expressed in diseased versus healthy tissues. Qualitative and quantitative biomarker measurement can help detect an illness at very early disease stages, and may be performed during routine examination of patient blood or tissue specimens. Identification of novel disease biomarkers also provides new molecular targets for drug development. Biomarkers can be prognostic or diagnostic, and their use in early disease detection can improve treatment success. For example, in melanoma, an aggressive skin-derived malignancy, protein expression changes during different stages can be used to identify high-risk patients who might benefit from aggressive therapy that would not be needed for other lower-risk patients [13]. Melanoma-associated protein biomarkers in patient sera provide important information regarding the likelihood of tumor metastasis and disease recurrence. The development of such patient-centric personalized medicine will improve disease diagnosis, and will guide treatment strategy selection to provide high therapeutic specificity and efficacy, while minimizing side effects [14].

\section{Public Awareness}

Modern healthcare is in need of improvement, and there is increasing demand for high quality, high-specificity treatments for diverse pathologies including neurodegenerative diseases, cardiovascular diseases, cancer, obesity, diabetes, and autoimmune diseases. The molecular research that underlies new treatment development is greatly propelled by material and informational support provided by biobanks [9]. It stands to reason that the public should be thoroughly informed regarding the purpose and operation of biobanks, and their important societal benefits. Public trust and confidence, which are vital for the success of the biobanking endeavor, can be cultivated by communication via seminars, genuine discussions among community members, health center meetings, workshops, interviews, internet social network forums, surveys, and other media sources (Figure 2). Success of biobanks depends on support from community members and public participation as volunteer/patient donors. The encouragement and support provided by existing donors and family physicians and doctors regarding biobanking are highly valuable for effective and sustainable participation of community members. However; religious and cultural beliefs mitigate against donating tissue specimens for biobanking or research among various ethnic populations. For example, Native Americans usually bury their body parts or use for other religious ceremonies [15]. Hence, to increase awareness and participation, additional sensitivity and effort toward understanding customs and beliefs is important when explaining biobanking to native populations.

As mentioned earlier, for identifying associations between a particular gene and a disease, large sample numbers need to be collected and processed. Thus enrollment of sufficient numbers of donors is crucial for the future of biobanks. A recent survey of more than 15,000 participants from 32 European countries has shown that about twothirds were unaware of the importance of biobanks [16]. Among these participants, the 'active group,' which discusses and frequently seeks information about biobanking, was limited to only $2 \%$ of respondents. Approximately $10 \%$ of respondents were unaware of the existence and utility of biobanks, but expressed interest in participating after understanding the purpose of biobanks. This study confirmed the need for development of more effective ways to educate the public about biobanks. In the United States, health agencies including the NIH are working on the development of National biobanks. Communication with the public regarding the issues and collecting feedback is being guided by The Genetics and Public Policy Center, John Hopkins University [17]. Similarly, to study the genetics of childhood diseases, a project involving the collection of blood samples from a large group of children $(100,000)$ has been recently launched by Philadelphia Children's Hospital, Pennsylvania, U.S.A. [18]. In the UK, during the development of the UK Biobank, the initial response rate of about 10\% resulted in enrollment of more than 100,000 donors from the first batch [19].

Because the biospecimens and the medical information associated with them are extremely valuable for assessing disease diagnosis, staging, and treatment, biobanks need to be expanded to provide larger sample numbers of greater diversity to researchers. To accomplish this, it is essential that public concerns regarding medical data confidentiality be addressed in detail. For biobank research, procurement of tissue samples and other biospecimens from donors is carried out only after obtaining informed consent, an absolute prerequisite. Documents disclosing the usage of biospecimens donated by individuals for current or future research and the related descriptive medical data are required by the medical institution's IRB (Institutional review board) for the approval of protocols. In addition to protecting the confidentiality of the records of the donors, the IRB continues to monitor the financial viability and performance of biobanks. IRBs also make sure that the documents and consent forms are understandable through proper communication with the volunteers/donors to avoid any linguistic barriers which limit the tissue/specimen donation. In the case of competent adults, the consent must be 'valid, full, and written' and signed by a research team member [20,21]. Consent forms containing the details of the purpose of research and explanations regarding the collection of health information from the patient should identify the experimental procedures, if any. Even if the purpose of research is not known at the time of collection of biospecimens for biobanking, federal regulations can be followed by describing the general types of planned future genetic research studies [22]. However, there are different possible solutions for 'future research' consent forms, as explained by Pawlikowski et al, based on surveys conducted in Polish biobanks, which include the 'broad consent' (Blanket Consent) form that allows the use of biospecimens and associated medical information for both immediate and future research. A 'partially restricted' consent allows biospecimen use for current research and for future research that is associated with the same patient, and finally, a 'specific consent' covers usage of tissues only for immediate research purposes [23-25]. In general, for irreversibly anonymized samples for which the 'sample source' cannot be identified, informed consent is not necessary as per international and regional regulations [26,27]. However, good communication between biobank personnel and donor is necessary to educate the donor/patient with respect to biobanking purposes, and thus, to make sound decisions as to the type of consent the donor is willing to provide (Figure 2). There are 'opt-in' and 'opt-out' patient recruitment processes for participating in biobanking. The 'opt-in' approach embodies the active participation of the donor in the study with routine use of patient data [28,29], whereas 'opt-out' require minimal involvement of individuals/donors in the research. 'Opt-in' consent forms describe the nature and purpose of research elaborately, while 'opt-out' forms generally provide a brief statement about the study. 


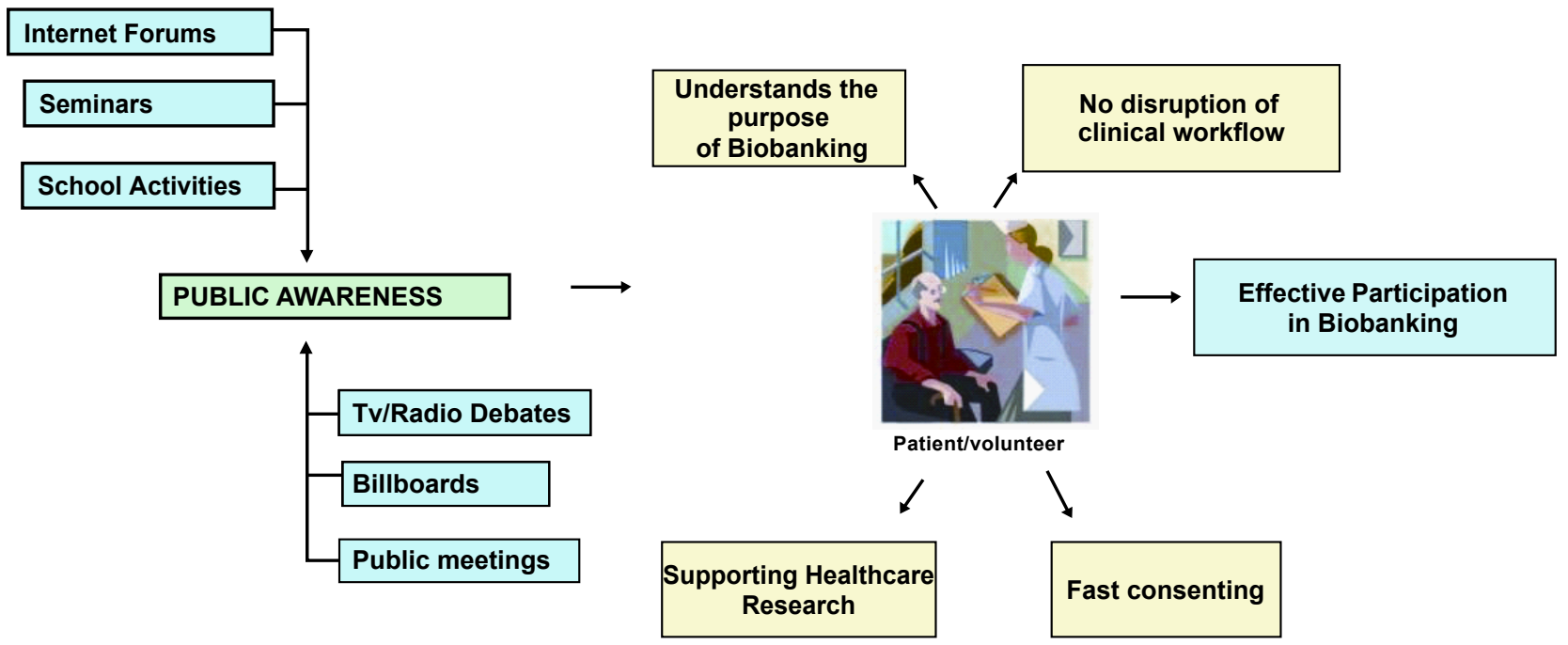

Figure 2: Public awareness about the purposes of biobanking is necessary for effective participation and fast consent from patients/volunteers in hospital-based biobanks.

However; there are certain challenges and issues to be addressed when inviting more public support and involvement to biobanking, mainly depending on the trust, privacy policies and data securities of the biobank. The scientists or physicians running biobanks should be trustworthy for the public and the language used while communicating should be transparent and fit for any layman. As explained by Gottweis et al, [30] reciprocity is another major factor that needs to be considered when people think that donations are not 'gifts' and they want something in return such as feed backs from the research, or annual updates about the contributions of biobanks to the healthcare research. However; for establishing a mutual respectful relationship between biobanks and the community, it is highly essential to make people understand the purposes of biobank and the advantages it provides to the community healthcare system.

A randomized study conducted at Hackensack University Medical Center, revealed general misconceptions among patients regarding the donation of tissues removed during surgical procedures and biopsies. The majority of patients believed that additional tissue samples would be procured from them or that the process would affect their clinical outcome once they signed an informed consent for tissue banking. Many expressed concern that if they did not consent to tissue banking, they would not obtain proper medical treatment. These misconceptions led patients to limit their donations to blood but not tissues. Many were afraid that they would become a "guinea pig" by participating in clinical trials, and would be required to donate an unlimited amount of tissue and blood for tests and tissue banking. Others worried about confidentiality and privacy of their health information.

Exam rooms are regularly used for patient education and signing the consent forms because there are typically no other designated areas available for this purpose; hence, the time taken to consent patients leads to a backlog of patients waiting to be seen by their physicians, causing major workflow problems. Educating a patient participating in research may require as much as 1 hour, although consenting pre-educated patients may require less than 5 minutes (Figure 2). Consenting involves explaining all aspects of tissue banking and providing detailed explanations of all consent form components.
Examples of these essential components include medical risks of tissue and blood donation, benefits, procedural details, financial disclosures, confidentiality guarantees, and patient rights. The Informed Consent document is between 7 pages (for tissue banking) and 20 pages (for a clinical trial), with the HIPAA form is an additional 2 pages.

Moreover, the physician is required to mention the tissue banking program to the patient before a nurse or research coordinator may approach the patient. Physicians are often concerned about the mental state of patients, who may be overwhelmed by their diagnosis and are wary of anything that may interfere with their treatment plan. Patents may be emotional and concerned about their upcoming procedure or treatment, and this becomes their main focus. Thus, it may be difficult to approach a patient with a request to participate in research at this time, which results in physicians often refraining from presenting the topic of biobanking.

\section{Banking of Samples and Traceability}

Generally, healthcare delivery institutions such as hospitals conduct patient-oriented sample banking, whereas University hospitals archive samples both for health purposes and for research. Biobanks archive samples solely for research purposes. Patient tissue samples can be freshly frozen or formalin fixed and paraffin embedded (FFPE) for archiving purposes based on standardized protocols (SOPs, standard operating procedures) [31], and blood samples can be fractionated into sera and cells, which are then preserved for easy researcher access. Biospecimens are stored according to their required preservation protocols [32]. For example, blood, plasma, serum, and DNA can be stored in $-80^{\circ} \mathrm{C}$ freezers, whereas tissues and cell lines can be preserved in liquid nitrogen freezers, thereby avoiding potential thermocycling damage. Sample contamination is always a concern, and extreme care should be taken for the preservation of non-fixed tissues or various body fluids. As explained by Eric J. Woods and Sreedhar Thirumala [33], biopreservation containers should have closure integrity and provide sample stability, and should provide easy access to the stored samples to avoid any type of sample contamination. Similarly, to minimize sample identification errors or misplacement, systems must 
be in place to ensure that the specific sample indeed belongs to the specific donor before the sample is supplied for research. Effective traceability of samples can be done using modern high-tech robotic procedures in the case of larger population biobanks, or through the use of donor genetic profiling systems. Because molecular or genetic profiling is currently expensive, this approach is not feasible for large donor populations. Hence, cost-effective models are in practice in DNA banks (e.g., DNA Bank of the University of the Basque Country, Spain) [34], which use small dried blood spots obtained from donors at the time of sampling, stored on 10-mm diameter paper or fabric discs at room temperature attached to an authentication form. Of note, the use of dried blood spots reduces the cost of storage, but this reduction of cost is only marginal as the majority of costs lie in the molecular profiling and other genetic testing. Moreover, secure maintenance of all relevant documents including consent forms, protocols, SOPs, and sample processing records, as well as location and storage details, is necessary for proper biobank management.

\section{Summary}

Biobanks may employ a population-based or disease-oriented format. Population biobanks are useful for assisting large cohort studies, and disease-oriented biobanks are essential for studying a particular disease. Disease-oriented biobanks preserve and archive patient samples for future analysis and identification of diseaserelevant biomarkers. Novel biomarker identification will ultimately help in developing personalized medical treatment regimens for 'lethal' diseases such as cancer, neurodegenerative diseases, and autoimmune diseases (Figure 1). Public awareness and appreciation of biobanking is essential for the success of biobanking projects. Public awareness campaigns are necessary to demystify the important roles of biobanks and tissue donation in propelling modern medicine. This will enhance public interest and participation, and will increase patient enrollment. Increased public awareness and knowledge will minimize the time required for patient education in the physician office and hospital setting (Figure 2). Public awareness and knowledge of biobanking can be achieved through discussions and debates in popular media, seminars, surveys, internet social forums, health center workshops, and library meetings where community members have easy access. These activities can be embedded in the education process as early as high school, and even more effectively, in college level courses where young adults realize the importance of biobanking in promoting biomedical research and understand that improvements in research will eventually lead to better healthcare. High school level classroom discussions of ethical components and benefits of biobanking will provide students with knowledge and awareness that can be shared outside the classroom as well as with parents. Similar education or discussion sessions on popular media such as social forums on the internet can promote biobanking awareness and educate the public. Educational advertisements on mass media such as magazines, newspapers, or through distribution of biobanking brochures at community events can ignite community-wide awareness of biobanking, and can increase awareness of the role of biobanking and research in improving healthcare. The inclusion of biobanking as a topic in healthcare-related seminars, and distribution of surveys at community sites can promote awareness of biobanking. Collaborative efforts of institutions, local government, and patient advocacy groups on issues pertaining to patient confidentiality and compliance can boost trust of the general population. Lastly, sustained funding and support must be provided for the long-term maintenance of biobanks. Future improvements in community health depend upon the development of personalized medical treatments through the identification and validation of disease-relevant biomarkers in banked donor specimens.

\section{Conclusions}

- Biobanking can promote awareness of health issues by the national and international community by archiving consented biospecimens for healthcare research.

- The biospecimens are used for identification of diagnostic and prognostic biomarkers for developing personalized treatments.

- Public awareness as to the purposes of biobanking are essential for their effective participation in biobanking, which can be achieved through popular media, seminars, public debates, surveys, and internet social forums.

- Education is crucial to avoid misconceptions among patients regarding donation of biospecimens for biobanking.

- Smaller biobanks (e.g., tissue banks) should solicit donations from sources such as academia, charitable organizations, individual donors, and Pharmaceutical companies. Government grants should support biobanking activities of community medical centers.

\section{Funding}

It is essential to have adequate funding available for the establishment of biobanks and to have additional ongoing grants/funding to cover operating costs and to guarantee long-term sustainability. Funding can be private, private-public or public depending upon the size and nature of the biobank. For example, larger population biobanks are typically supported by public funding (government and charitable organizations), while pharmaceutical biobanks are maintained by private funding. Lack of long-term funding impairs biobank functionality, which ultimately will have severe negative implications for community health. Adequate funding is prerequisite to biobank stability and expansion, unhindered sample provision to medical researchers, and the subsequent development of novel strategies to treat serious diseases. For instance, in the USA, the caHUB (cancer HUman Biobank, Bethesda, MD) was developed and supported by the National Cancer Institute for preserving high-quality human cancer tissue samples for medical research [35]. Similarly, the UK Biobank (UK Biobank Limited, United Kingdom) is funded by the Medical Research Council, Department of Health, and the Wellcome Trust. Smaller biobanks (tissue banks) may receive sustained funding from government sources via various grant mechanisms, but these institutions should solicit donations or promote realistic cost-recovery systems from various sources such as academia (sharing resources), charitable organizations, individua donors, and pharmaceutical companies (storage of clinical trial samples) to support biobank activities. Community hospitals have foundation offices that can promote philanthropic donations and promote campaigns that can support biobanking activities. All of these entities have vested interests in promoting biobank success, and ultimately, improving the health of current and future generations.

\section{References}

1. Gaffney EF, Madden D, Thomas GA (2012) The human side of cance biobanking. Methods Mol Biol 823: 59-77.

2. Webster JD, Simpson ER, Michalowski AM, Hoover SB, Simpson RM (2011) Quantifying histological features of cancer biospecimens for biobanking quality assurance using automated morphometric pattern recognition image analysis algorithms. J Biomol Tech 22: 108-118.

3. Hamburg MA, Collins FS (2010) The path to personalized medicine. N Eng J Med 363: 301-304

4. Forsberg JS, Hansson MG, Eriksson S (2009) Changing perspectives in biobank research: from individual rights to concerns about public health regarding the return of results. Eur J Hum Genet 17: 1544-1549.

5. Murtagh MJ, Demir I, Harris JR, Burton PR (2011) Realizing the promise of population biobanks: a new model for translation. Hum Genet 130: 333-345.

6. Riegman PH, Morente MM, Betsou F, de Blasio P, Geary P (2008) Biobanking for better healthcare. Mol Oncol 2: 213-222.

7. Hewitt RE (2011) Biobanking: the foundation of personalized medicine. Cur Opin Oncol 23: 112-119. 
Citation: Sarojini S, Goy A, Pecora A, Suh KS (2012) Proactive Biobanking to Improve Research and Health Care. J Tissue Sci Eng 3:116. doi:10.4172/2157-7552.1000116

Page 6 of 6

8. Hansson MG (2007) For the safety and benefit of current and future patients. Pathobiology 74: 198-205.

9. Vegvari A, Welinder C, Lindberg H, Fehniger TE, Marko-Varga G (2011) Biobank resources for future patient care: developments, principles and concepts. J Clin Bioinforma 1: 24.

10. Asslaber M, Zatloukal K (2007) Biobanks: transnational, European and global networks. Brief Functl genomic proteomic 6: 193-201.

11. Asslaber M, Abuja PM, Stark K, Eder J, Gottweis H, et al. (2007) The Genome Austria Tissue Bank (GATiB). Pathobiology 74: 251-258.

12. Vegvari A, Marko-Varga G (2010) Clinical protein science and bioanalytical mass spectrometry with an emphasis on lung cancer. Chem Rev 110: 32783298.

13. Sabel MS, Liu Y, Lubman DM (2011) Proteomics in melanoma biomarker discovery: great potential, many obstacles. Int J Proteomics 2011:181890.

14. Waldman SA, Terzic A (2011) Patient-centric clinical pharmacology advances the path to personalized medicine. Biomark Med 5: 697-700.

15. Vaught JB, Lockhart N, Thiel KS, Schneider JA (2007) Ethical, legal, and policy issues: dominating the biospecimen discussion. Cancer Epidemiol Biomarkers Prev 16: 2521-2523.

16. Gaskell G, Gottweis H (2011) Biobanks need publicity. Nature 471: 159-160

17. Goldman RE, Kingdon C, Wasser J, et al. (2008) Rhode Islanders' attitudes towards the development of a statewide genetic biobank. Personalized Medicine 5: 339-359.

18. Maschke KJ (2010) Biobanks: DNA and Research. ed NY2008.

19. Watts G (2007) UK Biobank gets $10 \%$ response rate as it starts recruiting volunteers. BMJ 334: 659 .

20. Sheikh AA (2004) Genetic research \& human biological samples: some legal and ethical considerations. Med Law 23: 897-912.

21. Auray-Blais C, Patenaude $\mathrm{J}$ (2006) A biobank management model applicable to biomedical research. BMC med Ethics 7: E4.

22. Beskow LM, Friedman JY, Hardy NC, Lin L, Weinfurt KP (2010) Developing a simplified consent form for biobanking. PloS One 5: e13302.

23. Pawlikowski J, Sak J, Marczewski K (2010) The analysis of the ethical, organizational and legal aspects of Polish biobanks activity. Eur J Public Health 20: 707-710.

24. Murphy J, Scott J, Kaufman D, Geller G, LeRoy L, et al. (2010) Public perspectives on informed consent for biobanking. Am J Public Health 99: 2128 2134.

25. Hall MA, King NM, Perdue LH, Hilner JE, Akolkar B, et al. (2010) Biobanking, consent, and commercialization in international genetics research: the Type 1 Diabetes Genetics Consortium. Clin Trials 7: S33-S45.

26. Knoppers BM (2005) Biobanking: international norms. J Law Med Ethics 33 : 7-14.

27. Salvaterra E, Lecchi L, Giovanelli S, Butti B, Bardella MT, et al. (2008) Banking together. A unified model of informed consent for biobanking. EMBO rep 9 : 307-313.

28. Junghans C, Feder G, Hemingway H, Timmis A, Jones M (2005) Recruiting patients to medical research: double blind randomised trial of "opt-in" versus "opt-out" strategies. BMJ 331: 940.

29. Simon CM, L'Heureux J, Murray JC, Winokur P, Weiner G et al. (2011) Active choice but not too active: public perspectives on biobank consent models. Genet Med 13: 821-831.

30. Gottweis H, Gaskell G, Starkbaum J (2011) Connecting the public with biobank research: reciprocity matters. Nat Rev Genet 12: 738-739.

31. Morente MM, Mager R, Alonso S, Pezzella F, Spatz A et al. (2006) TuBaFrost 2: Standardising tissue collection and quality control procedures for a European virtual frozen tissue bank network. Eur J Cancer 42: 2684-2691.

32. Hirtzlin I, Dubreuil C, Preaubert N, Duchier J, Jansen B et al. (2003) An empirical survey on biobanking of human genetic material and data in six EU countries. Eur J Hum Genet 11: 475-488.

33. Woods EJ, Thirumala S (2011) Packaging Considerations for Biopreservation Transfus Med Hemother 38: 149-156.

34. Cardoso S, Valverde L, Odriozola A, Elcoroaristizabal X, de Pancorbo MM (2010) Quality standards in Biobanking: authentication by genetic profiling of blood spots from donor's original sample. Eur J Hum Genet 18: 848-851.

35. Vaught J, Rogers J, Myers K, Lim MD, Lockhart N, et al. (2011) An NC perspective on creating sustainable biospecimen resources. J Natl Cancer Inst Monogr 2011: 1-7. 